\title{
Juxtaposition on Discrete Covid-19 Vaccines: For Rudimentary and Pivotal Cognizance
}

\author{
Pankaj Kisan Chatki ${ }^{* 1}$, Sana Tabassum ${ }^{2}$ \\ ${ }^{1}$ Analytical Research and Development Department, Slayback Pharma India LLP, Hyderabad, Telangana, India. \\ ${ }^{2}$ Pharmaceutical Analysis and Quality Assurance, Jawaharlal Nehru Technological University, Hyderabad, \\ Telangana, India.
}

\section{${ }^{*}$ Correspondence E-mail : pankajchatki22@gmail.com}

\begin{abstract}
This paper accomplishes an introduction to diverse types of vaccines against coronavirus. Many countries, researchers and pharmaceutical companies worked abundantly so that they could discover a safe and efficacious vaccine against corona virus. Thereby, vaccines were discovered on a fast track mode and companies registered for an emergency use authorization, so that it would be helpful to society during pandemic. Manufacturers and various regulatory units are working tirelessly to develop, manufacture and deploy safe and effective vaccines. Scanty information is added on divergent COVID vaccines encompassing information related to the developer, vaccine class, description, efficacy rate, dosing, storage, permitted age groups and authorizations issued.
\end{abstract}

Keywords: Coronavirus, Vaccine, Vaccine Class, Efficacy, Dosing and Authorizations.

\section{Introduction}

The novel corona virus-SARS-CoV-2 has led to the infectious disease COVID-19. It is a disease which mostly affects the respiratory system. Based on the severity of disease the symptoms vary from mild to severe illness. Symptoms appear within 2-14 days after the exposure of corona virus. Symptoms comprises of fever, cold, cough, chills, dyspnea, body ache, dysgeusia, anosmia, throat infections, diarrhoea etc. In this long run of the pandemic, vaccine is essential. (Baden et al., 2021; Pedro Folegatti et al., 2020).

\section{Review}

Distinct types of vaccines against coronavirus are listed below. The disparate vaccine class includes encapsulated mRNA, viral vector, inactivated virus, virus like particle and Plasmid DNA. Vaccines general names and trade names, developer name, and vaccine class related particulars are provided. Based on the published clinical trials data the number of doses to be administered with respective frequencies is given. Below provided information on the number of doses to be taken or administered and frequency is universal. Developers and manufacturers, based on the vaccines stock availability accordingly framed the dosing frequency, covering the safety and efficacy facets. A description is given appertaining to active and inactive ingredients involved in the respective vaccine. The storage conditions to be followed for a vaccine are given. Based on the data announced by the developer, efficacy of each listed vaccine is specified. Small information is added on the effectiveness of vaccines against other strains as per the declarations of the 
Int J Adv Life Sci Res. Volume 4(4)1-6

developer. Information on the major regulatory agencies, which approved Emergency Use Authorizations for the respective vaccine, is incorporated (Pfizer-BioNTech Sinopharm WHO 2021).

2021;

\section{Results}

Table : Juxtaposition on COVID-19 Vaccines

\begin{tabular}{|c|c|c|c|c|c|c|c|}
\hline $\begin{array}{c}\text { Vaccine Name } \\
\text { (Trade Names } \\
\text { \& Other } \\
\text { Names) } \\
\& \\
\text { Developed by }\end{array}$ & Vaccine Class & $\begin{array}{l}\text { Brief Description of } \\
\text { Active Ingredient }\end{array}$ & $\begin{array}{l}\text { Inactive Ingredients/ } \\
\text { Excipients } \\
\text { Composition }\end{array}$ & $\begin{array}{l}\text { Dosing and } \\
\text { Administration } \\
\text { Related } \\
\text { Information }\end{array}$ & $\begin{array}{c}\text { Efficacy Rate } \\
\text { after } \\
\text { complete } \\
\text { doses }\end{array}$ & Storage & $\begin{array}{c}\text { Major } \\
\text { Authorizations } \\
\text { and } \\
\text { (References) }\end{array}$ \\
\hline $\begin{array}{c}\text { BNT162b2/ } \\
\text { Comimatyl } \\
\text { Tozinameran } \\
\text { Pfizer- } \\
\text { BioNTech }\end{array}$ & $\begin{array}{c}\text { Encapsulated } \\
\text { mRNA }\end{array}$ & $\begin{array}{l}-0.3 \text { mL dose } \\
\text { comprise of } 30 \mathrm{mcg} \\
\text { of a nucleoside } \\
\text { mRNA encoded with } \\
\text { glycoprotein-viral } \\
\text { spike of SARS-CoV- } \\
2 \text {. } \\
\text { Prior to use to form } \\
\text { the vaccine, the vial } \\
\text { must be diluted with } \\
\text { sterie } 0.9 \% \text { Sodium } \\
\text { chloride- } 1.8 \mathrm{~mL} \text {. }\end{array}$ & 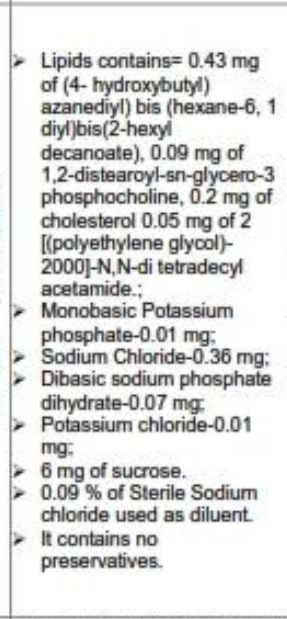 & $\begin{array}{l}\mathrm{V}=0.3 \mathrm{~mL} \\
\mathrm{~N}=2 \text { doses } \\
\mathrm{F}=21 \text { days } \\
\text { apart (i.e., } 3 \\
\text { weeks apart) } \\
\text { DF = Frozen } \\
\text { Suspension } \\
\text { ROA }=1 . \mathrm{M} \text {. }\end{array}$ & $\begin{array}{l}\text { Overall } \\
\text { efficacy } 95 \% \\
\text { against } \\
\text { Original } \\
\text { Strain } \\
79 \\
\text { effective } \% \\
\text { against delta } \\
\text { variant. }\end{array}$ & $\begin{array}{l}\text { Frozen } \\
\text { Vials: Before use } \\
\text { frozen vials to be } \\
\text { stored } \\
\text { temperature of at }-90 \\
{ }^{\circ} \mathrm{C} \text { to }-60^{\circ} \mathrm{C} \text { tall the } \\
\text { expiry date. Can be } \\
\text { stored at }-25^{\circ} \mathrm{C} \text { to }- \\
25{ }^{\circ} \mathrm{C} \text { for upto } 2 \\
\text { weeks. } \\
\text { Vials can be stored } \\
\text { at } 20^{\circ} \mathrm{C} \text { to } 8^{\circ} \mathrm{C} \text { for } \\
\text { up to } 5 \text { days. It can } \\
\text { be stored at Room } \\
\text { Ternperature, for } 30 \\
\text { minutes. } \\
2 \text { Undiluted } \\
\text { vials can be stored } \\
\text { at room temperature } \\
\text { upto } 2 \mathrm{hrs} \text {. } \\
\text { Viluted } \\
\text { Vials to be placed at } \\
2^{\circ} \mathrm{C} \text { to } 25^{\circ} \mathrm{C} \text { and } \\
\text { should be used } \\
\text { within } 6 \text { hrs. from } \\
\text { dilution time. }\end{array}$ & $\begin{array}{l}\text { FDA and WHO } \\
\text { issued EUA. } \\
\text { PAG = } 12 \text { years } \\
\text { and older } \\
\text { Aiming to } \\
\text { implement booster } \\
\text { dose for emerging } \\
\text { variant. } \\
\text { (Fernando Polack } \\
\text { et al., 2021: } \\
\text { Edward W ot al., } \\
2020 ; \text { Pfizer- } \\
\text { BioNTech 2021) }\end{array}$ \\
\hline $\begin{array}{l}\text { mRNA-1273/ } \\
\text { Elasomeran' } \\
\text { Spikevax } \\
\text { Moderna }\end{array}$ & $\begin{array}{l}\text { Encapsulated } \\
\text { mRNA }\end{array}$ & $\begin{array}{l}\text { The vaccine is } \\
\text { designed such that } \\
\text { each } 0.5 \text { mL } \\
\text { comprises of } \\
\text { mucleoside modified } \\
\text { mRNA(100 mcg) } \\
\text { encoded with spike } \\
\text { glycoprotein of } \\
\text { SARS-CoV-2(pre } \\
\text { fusion stabilized } \\
\text { form) }\end{array}$ & $\begin{array}{l}\text { - It contains lipid content of } \\
1.93 \mathrm{mg} \text { which includes } \\
\text { PEG-2000, dimyristoyl } \\
\text { glycerol, SM-102, 1,2- } \\
\text { distearoyl-sn glycero-3- } \\
\text { phospho choline and } \\
\text { cholesterol; } \\
\text { - Tromethamine HCl (1.18 } \\
\text { mg); } \\
\text { - } 0.043 \mathrm{mg} \text { of Acetic acid; } \\
\text { - Tromethamine (0.31 mg); } \\
\text { - } 0.20 \mathrm{mg} \text { Sodium acetate } \\
3 \mathrm{H} 2 \mathrm{O} \text {; } \\
\text { - } 43.5 \mathrm{mg} \text { of Sucrose. } \\
\text { - Contains no preservatives. }\end{array}$ & $\begin{array}{l}\mathrm{V}=0.5 \mathrm{~mL} \\
\mathrm{~N}=2 \text { doses } \\
\mathrm{F}=1 \text { month } \\
\text { apart. } \\
\mathrm{DF}=\text { Frozen } \\
\text { suspension } \\
\mathrm{ROA}=1 . \mathrm{M} .\end{array}$ & $\begin{array}{l}\text { Overall } \\
\text { efficacy } \\
\text { proved to be } \\
94.1 \% \text { (OS) }\end{array}$ & $\begin{array}{l}\text { Multiple- } \\
\text { dose vials - stored } \\
\text { frozen between - } \\
50^{\circ} \mathrm{C} \text { to }-15^{\circ} \mathrm{C} \text {. } \\
2 \text { It must be } \\
\text { not stored at or } \\
\text { below }-50^{\circ} \mathrm{C} \text { and on } \\
\text { dry ice. Can be } \\
\text { stored at } 2^{\circ} \text { to } 8^{\circ} \mathrm{C} \\
\text { upto } 30 \text { days. } \\
\text { stored at } 8^{\circ} \text { to } 25^{\circ} \mathrm{C} \\
\text { till } 24 \text { hrs only. } \\
\text { withdrawal to be } \\
\text { placed within } 2^{\circ} \text { to } \\
25^{\circ} \mathrm{C} \text {. After the } \\
\text { puncture to be } \\
\text { discarded after } 12 \\
\text { hrs. Once } \\
\text { thawed, not to be } \\
\text { refreeze. } \\
\text { vials can be placed } \\
\text { at room light } \\
\text { conditions. }\end{array}$ & $\begin{array}{l}\text { US FDA and WHO } \\
\text { issued EUA. } \\
\text { PAG = Authorized } \\
\text { for } 12 \text { years and } \\
\text { older } \\
\text { (Baden et al., 2021; } \\
\text { Lisa Jackson et al., } \\
2020 \text {; Moderna } \\
\text { Vaccine- Fact } \\
\text { Sheet 2021). }\end{array}$ \\
\hline $\begin{array}{l}\text { Covishield/ } \\
\text { ChAdOx1-S/ } \\
\text { AZD1222/ } \\
\text { Vaxzevria } \\
\text { Oxford } \\
\text { University and } \\
\text { AstraZeneca. }\end{array}$ & Viral Vector & $\begin{array}{l}\text { - Vaccine is designed } \\
\text { such that it } \\
\text { constitutes } \\
\text { recombinant of } \\
\text { replication of } \\
\text { adenovirus virus(from } \\
\text { Chimpanzee) } \\
\text { encoded with the } \\
\text { spike glycoprotein. } \\
\text { - it is produced by } \\
\text { rDNA technology by } \\
\text { genetically modfying } \\
\text { the human embryonic } \\
\text { cells. }\end{array}$ & $\begin{array}{l}\text { Inactive ingredients } \\
\text { includes } \mathrm{MgCl} \\
\text { hexahydrate, Disodium } \\
\text { EDTA, Histidine, Histidine } \\
\text { HCl monohydrate, Ethanol; } \\
\text { Polysorbate } 80 ; \mathrm{NaCl} \text {, } \\
\text { Sucrose; and Sterile water. } \\
\text { - It is preservative free. }\end{array}$ & $\begin{array}{l}\mathrm{V}=0.5 \mathrm{~mL} \\
\mathrm{~N}=2 \text { doses } \\
\mathrm{F}=\text { Between } 4 \\
\text { to } 12 \text { weeks } \\
\text { apart, as per } \\
\text { WHO } 8 \text { to } 12 \\
\text { weeks apart. } \\
\mathrm{DF}=\text { Solution } \\
\mathrm{ROA}=\text { I.M. }\end{array}$ & $\begin{array}{l}\text { Overall } \\
\text { efficacy } 82 \% \\
\text { (OS) } \\
-61 \% \\
\text { effective } \\
\text { against delta } \\
\text { variant. }\end{array}$ & $\begin{array}{l}\text { Multidose } \\
\text { unopened vials to } \\
\text { be stored at } \\
\text { refrigerator } \\
\text { conditions i.e., at }+2 \\
\text { to }+8^{\circ} \mathrm{C} \text { for upto } 6 \\
\text { months. Not to be } \\
\text { freezed. Opened } \\
\text { vials can be stored } \\
\text { at room } \\
\text { temperature(for } \\
6 \mathrm{hrs} \text { ); or between } \\
+2 \text { to }+8^{\circ} \mathrm{C} \text { (for } 48 \\
\text { hrs.).No No } \\
\text { reconstitution is } \\
\text { required. }\end{array}$ & $\begin{array}{l}\text { EMA, WHO issued } \\
\text { EUA. } \\
\text { PAG = Authorized } \\
\text { for } 18 \text { years and } \\
\text { older } \\
\text { (Merryn a, b et al., } \\
2021 ; \text { Pedro } \\
\text { Folegatti et al., } \\
2020 ; \text { Astrazeneca } \\
\text { Canada Inc. 2021; } \\
\text { EMA Vaxzevria } \\
\text { 2021; EMA } \\
\text { AstraZeneca 2021). }\end{array}$ \\
\hline $\begin{array}{l}\text { JNJ-78436735 } \\
\text { Also known as, } \\
\text { Ad26.COV2.S. } \\
\text { Janssen } \\
\text { Vaocines- } \\
\text { Johnson and } \\
\text { Johnson }\end{array}$ & Viral Vector & $\begin{array}{l}\text { This vaccine carries } \\
\text { replicated } \\
\text { incompetent } \\
\text { recombinant } \\
\text { Adenovirus 26, } \\
\text { expressing spike } \\
\text { protein with stabilized } \\
\text { conformation. } \\
\text { Each dose is } \\
\text { formulated such that } \\
\text { it contains 5*1010 } \\
\text { virus particles. }\end{array}$ & $\begin{array}{l}\text { Inactive ingredients } \\
\text { comprises of } 2 \mathrm{mg} \text { of } \\
\text { Trisodium } 2 \mathrm{H} 2 \mathrm{O} ; 2 \mathrm{mg} \text { of } \\
\text { Ethanol; } 0.14 \mathrm{mg} \text { of Citric } \\
\text { acid } \mathrm{H} 2 \mathrm{O} ; 2 \mathrm{mg} \text { of } \mathrm{NaCl} \text {; } \\
0.16 \mathrm{mg} \text { of Polysorbate-80 } \\
\text { and } 25.5 \mathrm{mg} \text { of } 2 \text { - } \\
\text { hydroxypropyl- } \beta \text { - It is } \\
\text { cyclodextrin. it is } \\
\text { preservative free. }\end{array}$ & $\begin{array}{l}\mathrm{V}=0.5 \mathrm{~mL} \\
\mathrm{~N}=1 \text { dose } \\
\mathrm{DF}= \\
\text { Suspension } \\
\mathrm{ROA}=\mathrm{L} . \mathrm{M} .\end{array}$ & 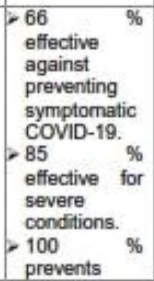 & 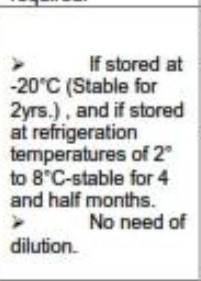 & $\begin{array}{l}\text { US FDA and EMA } \\
\text { issued EUA. } \\
\text { PAG = Authorized } \\
\text { for } 18 \text { years and } \\
\text { older. } \\
\text { Based on clinical } \\
\text { trials it is evident } \\
\text { that a single shot of } \\
\text { this vaccine } \\
\text { generated strong } \\
\text { effectiveness even } \\
\text { with the Delta }\end{array}$ \\
\hline
\end{tabular}




\begin{tabular}{|c|c|c|c|c|c|c|c|}
\hline & & & & & $\begin{array}{l}\text { hospitalizatio } \\
\mathrm{n} \text { or death. }\end{array}$ & & $\begin{array}{l}\text { variant and viral } \\
\text { variants of corona } \\
\text { virus. } \\
\text { (Jerald a, b et al., } \\
\text { 2021; Janssen } \\
\text { COViD-19 Vaocine } \\
\text { 2021). }\end{array}$ \\
\hline $\begin{array}{l}\text { Sputnik V } \\
\text { Also known as } \\
\text { Gam-Covid } \\
\text { Vac. } \\
\text { Gamaleya- } \\
\text { Research } \\
\text { Institute of } \\
\text { Epidemiology } \\
\text { \& Microbiology }\end{array}$ & $\begin{array}{c}\text { It is a } \\
\text { Combined } \\
\text { Viral Vector } \\
\text { based } \\
\text { heterologous } \\
\text { vaccine } \\
\text { (Adenovirus } \\
\text { viral vector are } \\
\text { rAd26 and } \\
\text { rAd5) }\end{array}$ & $\begin{array}{l}\text { Manufactured by } \\
\text { biotechnology, } \\
\text { without utilizing } \\
\text { SARS-Cov-2 virus. } \\
\text { Component } \\
\text { incorporates } \\
\text { recombinant } \\
\text { adenoviral vector of a } \\
\text { serotype 26 human } \\
\text { adenovirus with a } \\
\text { protein S gene of } \\
\text { SARS-CoV-2. } \\
\text { Component II } \\
\text { incorporates serotype } \\
5 \text { human adenoviral } \\
\text { vector with a protein } \\
\text { S gene of SARS- } \\
\text { CoV-2. dose=each } \\
\text { Per cormponent includes } \\
\text { (1.0t0.5) x 1011 } \\
\text { particles. }\end{array}$ & 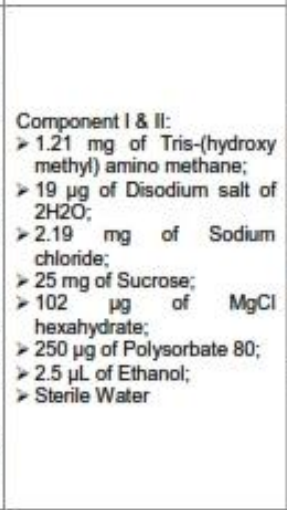 & $\begin{array}{l}\mathrm{V}=0.5 \mathrm{~mL} \\
\mathrm{~N}=2 \text { doses } \\
\mathrm{F}=3 \text { weeks } \\
\text { apart. } \\
\text { Dose } 1 \text { (i.e., Day } \\
0 \text { ) }-0.5 \mathrm{~mL} \text { of } \\
\text { component-1. } \\
\text { and } \\
\text { Dose } 2 \text { (i.e., on } \\
\text { Day } 21 \text { ) } 0.5 \mathrm{~mL} \\
\text { of component-1I } \\
\text { DF }=\text { Solution } \\
\text { ROA = I.M. }\end{array}$ & $\begin{array}{l}\text { - } 91.6 \\
\text { effective } \\
\text { against } \\
\text { Coronavirus } \\
\text { (OS) } \\
\text { - } 83 \% \\
\text { effective } \\
\text { against delta } \\
\text { variant }\end{array}$ & $\begin{array}{l}\text { Store at a } \\
\text { temperature NMT - } \\
18^{\circ} \mathrm{C} \text { in a dark } \\
\text { place. Thawed } \\
\text { vial to be used } \\
\text { within } 2 \text { hrs. } \\
\text { dilution. No need of } \\
\text { re-freezed, once the } \\
\text { vial is thawed. }\end{array}$ & $\begin{array}{l}\text { US FDA and EMA } \\
\text { issued EUA. } \\
\text { PAG = Authorized } \\
\text { for } 18 \text { years and } \\
\text { older. } \\
\text { (Denis Logunov et } \\
\text { al.,2020; Denis } \\
\text { Logunov ot } \\
\text { al.,2020; Ministry of } \\
\text { Health - Russian } \\
\text { Federation 2020; } \\
\text { Dr. Reddy's } \\
\text { SPUTNIK V Gam- } \\
\text { COVD-Vac 2021). }\end{array}$ \\
\hline $\begin{array}{l}\text { Covaxin; } \\
\text { BBV152 } \\
\text { Bharat Biotech } \\
\text { and } \\
\text { ICMR-Indian } \\
\text { Council of } \\
\text { Medical } \\
\text { Research }\end{array}$ & $\begin{array}{l}\text { Whole Virus- } \\
\text { Inactivated }\end{array}$ & $\begin{array}{l}\text { Single solitary dose } \\
\text { comprise of } 6 \text { jg of } \\
\text { inactivated SARS- } \\
\text { CoV-2 antigen-whole } \\
\text { virion (Strain: NIV- } \\
2020-770 \text { ) and it is } \\
\text { incorporated with a } \\
7 / 8 \text { agonist molecule } \\
\text { adsorhed on to alum- } \\
\text { toll ilke receptor }\end{array}$ & $\begin{array}{l}\text { Inactive ingredients include } \\
15 \mathrm{\mu g} \text { of TLR } 7 / 8 \text { imidazo } \\
\text { quinolinone agonist ; } 250 \mu \mathrm{\mu g} \\
\text { of gel-Aluminum hydroxide } \\
-2.5 \mathrm{mg} \text { of } 2-\text { phenoxy } \\
\text { ethanol and } 0.5 \mathrm{~mL} \text { saline } \\
\text { Phosphate buffer. }\end{array}$ & $\begin{array}{l}\mathrm{V}=0.5 \mathrm{~mL} \\
\mathrm{~N}=2 \text { doses } \\
\mathrm{F}=28 \text { days } \\
\text { apart } \\
\mathrm{DF}=\text { Solution } \\
\mathrm{ROA}= \\
\text { I.M. }\end{array}$ & $\begin{array}{l}93.4 \% \text { (OS) } \\
\text { effective for } \\
\text { severe } \\
\text { cases. } \\
\text { Overall } \\
\text { vaccine } \\
\text { efficacy is = } \\
77.8 \% \\
\text { against } \\
\text { symptomatic } \\
\text { infections. } \\
65.2 \\
\text { protection } \\
\text { against } \\
\text { infection with } \\
\text { delta variant. }\end{array}$ & $\begin{array}{l}\text { To be } \\
\text { stored at }+2^{\circ} \text { to }+8 \\
\text { "C. Not to be } \\
\text { freezed, if done so } \\
\text { to be discarded. } \\
\text { vials must be used } \\
\text { within } 6 \mathrm{hrs} \text {, if kept } \\
\text { at } 2 \text { to } 8{ }^{\circ} \mathrm{C} \text {. }\end{array}$ & $\begin{array}{l}\text { CDSCO issued } \\
\text { EUA. } \\
\text { PAG = Authorized } \\
\text { for } 18 \text { years and } \\
\text { older. } \\
\text { Clinical trials: } \\
\text { Ongoing for age } \\
\text { group 2-12 years. } \\
\text { Efficacy data } \\
\text { demonstrates } \\
\text { protection against } \\
\text { delta variant. to } \\
\text { Proven a alpha, } \\
\text { neutralize a beta, gamma, zeta } \\
\text { delta, kappa } \\
\text { variant. } \\
\text { (Raches a, b et al., } \\
\text { 2021; Bharat } \\
\text { Biotech } \\
\text { International Ltd } \\
\text { 2021). }\end{array}$ \\
\hline $\begin{array}{l}\text { CoronaVac } \\
\text { SinoVac } \\
\text { Biotech Lid. }\end{array}$ & $\begin{array}{l}\text { Inactivated } \\
\text { Virus }\end{array}$ & $\begin{array}{l}\text { Each dose (0.5mL) } \\
\text { contains } 600 \text { SU of } \\
\text { inactivated corona } \\
\text { virus as antigen. } \\
\text { The virus strain used } \\
\text { is CZ02, this is } \\
\text { inoculated into Vero } \\
\text { cell of African green } \\
\text { monkey kidney cell, } \\
\text { which is then } \\
\text { inactivated and finally } \\
\text { adsorbed by } \\
\text { aluminum hydroxide. }\end{array}$ & $\begin{array}{l}\text { - Inactive ingredients contains } \\
\text { NaCl, Disodium hydrogen } \\
12 \mathrm{H} 20, \mathrm{Na} \text { dihydrogen } \\
\text { phosphate H20, Al } \\
\text { hydroxide (as adjuvant); and } \\
\text { sterile Water. } \\
\text { - It is free of preservatives. }\end{array}$ & $\begin{array}{l}\mathrm{V}=0.5 \mathrm{~mL} \\
\mathrm{~N}=2 \text { doses } \\
\mathrm{F}=14-28 \text { days } \\
\text { apart } \\
\text { DF = } \\
\text { Suspension } \\
\text { ROA }= \\
\text { I.M. }\end{array}$ & $\begin{array}{l}\text { Overall } \\
\text { efficacy } 83.5 \\
\% \text {. } \\
65.9 \% \\
\text { effective for } \\
\text { the } \\
\text { prevention. } \\
-87.5 \\
\text { effective for } \\
\text { the } \\
\text { prevention of } \\
\text { hospitalizatio } \\
\text { n. } \\
90.3 \% \\
\text { effective } \\
\text { against ICU } \\
\text { circumstance } \\
\text { s. } \\
\text { - } 86.3 \% \text { for the } \\
\text { prevention of } \\
\text { death. }\end{array}$ & $\begin{array}{l}\text { stored at a } a \\
\text { temperature of }+2-8 \\
{ }^{\circ} \mathrm{C} \text { and must be } \\
\text { protected from light. }\end{array}$ & $\begin{array}{l}\text { WHO issued EUA. } \\
\text { PAG = Authorized } \\
\text { for } 18 \text { years and } \\
\text { older. } \\
\text { (Alejandro et al., } \\
2021 ; \text { Mine Durusu et } \\
\text { al., 2021; Sinowac } \\
\text { Life Sciences 2021). }\end{array}$ \\
\hline $\begin{array}{l}\text { BBIBP-CorV } \\
\text { Sinopharm's- } \\
\text { Beijing Institute } \\
\text { of Biological } \\
\text { Pdits Co. }\end{array}$ & $\begin{array}{l}\text { Inactivated } \\
\text { Virus }\end{array}$ & $\begin{array}{l}\text { - It is formulated as } \\
\text { vero cell based- } \\
\text { adjuvanted with } \\
\text { aluminium hydroxide- } \\
\text { adjuvanted and } \beta \text { - } \\
\text { propiolactone. } \\
\text { - it is an inactivated } \\
\text { vaccine based on the } \\
\text { strain-19nCOV-CDC- } \\
\text { TAN-HBO2 (HB02 } \\
\text { strain). } \\
\text { Each dose is } \\
\text { contributes to } 6.5 \text { U } \\
\text { or } 4 \mu g \text { of inactivated } \\
\text { virus antigen. }\end{array}$ & $\begin{array}{l}\text { Inactive } \\
\text { includes Al hydroxide } \\
\text { adjuvant in phosphate } \\
\text { buffered saline (PBS). } \\
\text { - PBS contains disodium } \\
\text { hydrogen phosphate } 12 \\
\text { H20, NaCl and } \mathrm{Na} \\
\text { dihydrogen phosphate. }\end{array}$ & $\begin{array}{l}\mathrm{V}=0.5 \mathrm{~mL} \\
\mathrm{~N}=2 \text { doses } \\
\mathrm{DF}= \\
\text { Suspension } \\
\mathrm{F}=21 \text { days } \\
\text { apart } \\
\text { ROA }=1 . \mathrm{M} .\end{array}$ & $\begin{array}{l}-74 \% \text { against } \\
\text { asymptomati } \\
\text { c and } \\
\text { symptomatic } \\
\text { cases. } \\
\text { Nearly } 100 \% \\
\text { against } \\
\text { severe } \\
\text { conditions. }\end{array}$ & $\begin{array}{l}\text { To be } \\
\text { stored at } 2-8 \text { "C, } \\
\text { protected from light. } \\
\text { frozen. Not to be }\end{array}$ & $\begin{array}{l}\text { WHO issued EUA. } \\
\text { General PAG = } \\
\text { Authorized for } 18 \text { to } \\
60 \text { years. } \\
\text { UAE and China } \\
\text { approved the EUA for } \\
3-17 \text { aged } \\
\text { adolescents and for } \\
\text { children (Sinopharm } \\
\text { WHO 2021; Shengli } \\
\text { et al., 2020). }\end{array}$ \\
\hline $\begin{array}{c}\text { Covovax, also } \\
\text { known as } \\
\text { NVX-CoV2373 } \\
\text { Novavax, Coali }\end{array}$ & $\begin{array}{l}\text { Virus like } \\
\text { particle }\end{array}$ & $\begin{array}{lr}\text { Per dose formulated } \\
\text { such that } & \text { it } \\
\text { incorporates } & \text { a } \\
\text { recombinant } & \\
\text { nanoparticle } & \text { spike }\end{array}$ & $\begin{array}{l}\text { Inactive substances are } \\
\text { NaCl, Dibasic } 7 \text { H2O, } \\
\text { Sodium } \\
\text { monobasic } \mathrm{H} 2 \mathrm{O} \text {, Monphate, } \\
\text { Potassium }\end{array}$ & $\begin{array}{l}\mathrm{V}=0.5 \mathrm{~mL} \\
\mathrm{~N}=2 \text { doses } \\
\mathrm{F}=21 \text { days } \\
\text { apart } \\
\mathrm{DF}=\end{array}$ & $\begin{array}{l}\text { Overall } \\
\text { efficacy of } \\
89.7 \% \text { after } \\
\text { the } \\
\text { dose. final } \\
\end{array}$ & $\begin{array}{l}\text { Can be } \\
\text { stored for } 6 \text { months } \\
\text { if stored between } 2- \\
8^{\circ} \mathrm{C} \text { and for } 2 \text { yrs. If } \\
\text { stored at }-20^{\circ} \mathrm{C} \text {. }\end{array}$ & $\begin{array}{l}\text { EMA issued EUA. } \\
\text { General PAG = } \\
\text { Authorized for } 18 \text { to } \\
60 \text { years. } \\
\text { (Paul Heath et }\end{array}$ \\
\hline
\end{tabular}


Int J Adv Life Sci Res. Volume 4(4)1-6

\begin{tabular}{|c|c|c|c|c|c|c|c|}
\hline $\begin{array}{l}\text { tion for } \\
\text { Epidemic } \\
\text { Preparedness } \\
\text { Innovations }\end{array}$ & & $\begin{array}{l}\text { protein of } 5 \mu g \text { and } \\
\text { Matrix-M adjuvant of } \\
50 \mu g\end{array}$ & $\begin{array}{l}\text { Polysorbate } 80, \mathrm{KCl} \text {, Water, } \\
\mathrm{NaOH} \text {, matrix adjuvant and } \\
\mathrm{HCl} \text {. } \\
\text { - it does not contain any } \\
\text { preservatives. }\end{array}$ & $\begin{array}{l}\text { Suspension } \\
\text { ROA }=1 . \mathrm{M} .\end{array}$ & \begin{tabular}{|l}
$-86.3 \%$ \\
against the \\
variant of \\
B.1.1.7. \\
$-96.4 \%$ w.r.t \\
non-B.1.1.7 \\
variants.
\end{tabular} & & $\begin{array}{l}\text { al.2021; } \\
\text { 2021). }\end{array}$ \\
\hline $\begin{array}{l}\text { ZyCov-D } \\
\text { Zydus } \\
\text { Cadila(Cadila } \\
\text { Healthcare } \\
\text { Limited) }\end{array}$ & $\begin{array}{l}\text { World's first } \\
\text { Plasmid DNA } \\
\text { Vaccine }\end{array}$ & $\begin{array}{l}\text { Vaccine formulated } \\
\text { such that, the } \\
\text { Plasmid DNA } \\
\text { incorporates of the } \\
\text { spike S gene corona } \\
\text { virus(SARS Co-V) } \\
\text { accompanying gene } \\
\text { coding for generation } \\
\text { of a signal peptide. } \\
\text { The spike S } \\
\text { comprised of receptor } \\
\text { which binds to the } \\
\text { human and ACE- } \\
\text { angiotensin } \\
\text { converting Enzyme } 2 \\
\text { receptor. } \\
\text { During clinical study it } \\
\text { was confirmed that } \\
\text { this vaccine mediates } \\
\text { the entry of virus } \\
\text { inside the cell. } \\
\text { Study doses includes } \\
\text { intradermal route at } \\
\text { 1mg. } 2 \text { mg dose. }\end{array}$ & $\begin{array}{l}\text { Developer has not published } \\
\text { or revealed any information } \\
\text { related to the inactive } \\
\text { ingredients still. }\end{array}$ & $\begin{array}{l}\text { Dose = Every } \\
\text { dose is } 2 \text { mg } \\
\text { split into two } \\
\text { parts, } \\
\text { administered at } \\
\text { two separate } \\
\text { sites. } \\
\mathrm{N}=3 \text { doses } \\
\mathrm{F}=\text { Day } 0,28 \text { th } \\
\text { 56th day } \\
\text { DF } \\
\text { Suspension } \\
\text { ROA = } \\
\text { It } \\
\text { administered is } \\
\text { using a needle } \\
\text { free applicator- } \\
\text { The PharmaJet } \\
\text { This type of } \\
\text { administration is } \\
\text { confirmed to as } \\
\text { painless } \\
\text { intradermal } \\
\text { route. }\end{array}$ & $\begin{array}{l}-66.6 \% \\
\text { efficacious } \\
\text { against } \\
\text { symptomatic } \\
\text { cases. } \\
100 \% \\
\text { effective for } \\
\text { moderate to } \\
\text { severe } \\
\text { disease } \\
\text { - Efficacy } \\
\text { results data } \\
\text { is reported } \\
\text { for interim } \\
\text { analysis of } \\
\text { its phase } 3 \\
\text { study trial. }\end{array}$ & $\begin{array}{l}\text { During } \\
\text { study it was stored } \\
\text { at } 2-8^{\circ} \text { C. } \\
\text { when stored at } 25 \\
\text { "C it was found to } \\
\text { be stable for at least } \\
3 \text { months. }\end{array}$ & $\begin{array}{l}\text { DCGI issued EUA. } \\
\text { Phase-I and II trials } \\
\text { showed its safety in } \\
\text { all age groups. } \\
\text { To be approved } \\
\text { PAG = Authorized } \\
\text { for adults age } \\
\text { group adolescents 12-18 } \\
\text { adoles and } \\
\text { years age group. } \\
\text { (Taufik et al., 2021; } \\
\text { Ayan et al., 2021; } \\
\text { Zydus Cadila 2021). }\end{array}$ \\
\hline
\end{tabular}

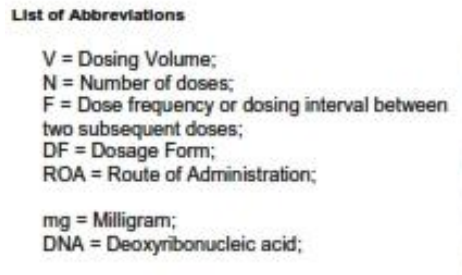

\section{Discussion}

Many divergent laboratories and Pharma companies have developed effective vaccines against COVID-19. The strive done by researchers and regulatory agencies is boundless. Though, throughout the world pandemic was there, they didn't lose their hope and made eminent efforts in developing and manufacturing vaccines. Continuous attempts are being made by developers to develop vaccine that could be effective against all strains of corona virus. After the defined doses series, now even booster shots of vaccines are also being given for certain age groups. Majority of vaccines are administered via intramuscular injection. Many of the vaccine candidates have surpassed Phase-III clinical trials. Apart from efficacy rate of vaccines, safety of vaccines is also equally given significant and scrutinized. Even though, vaccines have stabilized the mortality rates, we as humans also must adhere to Covid protocols to the extent possible, so that we can avoid another pandemic situation. Still studies are going on regarding administration of vaccines to infants, adolescents and pregnant womens. Ultimately the global endeavour to generate safe and effective vaccines is bearing fruit.

\author{
$\mathrm{OS}=$ Original Strain; \\ EUA = Emergency Use Authorization; \\ US FDA $=$ United States Food and Drug Administration; \\ PAG = Permitted Age group for usage; \\ mRNA = Messenger Ribonucleic Acict \\ COVID = Coronavirus Disease.
}

\section{Conclusion}

Various mentioned vaccines against coronavirus were developed on fast track modes after the outbreak of coronavirus. Every respective vaccine was subjected for estimating the safety and efficacy. Upon the approvals of emergency use authorizations, developed vaccines. Many of the vaccines are now being administered to humans in various countries. Existing data and facts suggests that the vaccines discovered may be contributory in saving individuals and even for diminishing the disease expansion during pandemic and post-pandemic. This article assists various researchers, manufacturers, and scientists to crisply study on various types of COVID vaccines. Provided juxtaposition, aids the reader to perceive about the developer, class of vaccine, active and inactive ingredients involved, efficacy, storage conditions, permitted age groups and authorizations approved on various types of vaccines against coronavirus.

\section{Acknowledgments}

The authors are thankful to the authorities of Slayback Pharma India LLP and Jawaharlal Nehru Technological University, Hyderabad, Telangana, India for giving necessary permission to carry over the review work. 


\section{Conflicts of Interest}

The authors declare no conflict of interest.

\section{References}

Alejandro, J., Eduardo Undurraga, A,, Cecilia, González., Fabio, P., Tomás, F., et al. (2021). Effectiveness of an Inactivated SARS-CoV-2 Vaccine in Chile. The New England Journal of Medicine, $\quad 385, \quad 875-884$. https://doi.org/10.1056/NEJMoa2107715

Astrazeneca Canada Inc. (2021, September 15). Product Monograph Including Patient Medication Information Astrazeneca Covid-19 Vaccine (Chadox1-S [Recombinant]). Retrieved September 20, 2021, from https://covidvaccine.canada.ca/info/pdf/astrazeneca-covid-19vaccine-pm-en.pdf

Ayan, D., Chozhavel Rajanathan, T. M., Harish, C., Hari Pericherla, P. R., Sanjeev, K., et al. (2021). Immunogenic potential of DNA vaccine candidate, ZyCoV-D against SARS-CoV-2 in animal models. Vaccine, $\quad 39 \quad$ (30), $\quad 4108-4116$. https://doi.org/10.1016/j.vaccine.2021.05.098

Baden, L. R., El Sahly, H. M., Essink, B,, Kotloff, K., Frey, S., Novak, R., et al. (2021). Efficacy and Safety of the mRNA-1273 SARS-CoV-2 Vaccine. The New England Journal of Medicine, 384(5), 403416. https://doi.org/10.1056/NEJMoa2035389

Bharat Biotech International Ltd.(2021, January 15). Covaxin- Summary of Product Characteristics. Retrieved August 29, 2021, from https://cdsco.gov.in/opencms/export/sites/CDSCO WEB/en/COVAXIN-SMPC -BBIL.pdf

Denis Logunov, Y., Inna Dolzhikova, V., Olga Zubkova, V., Amir, Tukhvatullin I., Dmitry Shcheblyakov, V., et al. (2020). Safety and immunogenicity of an rAd26 and rAd5 vector-based heterologous prime-boost COVID-19 vaccine in two formulations: two open, non-randomised phase $1 / 2$ studies from Russia. The Lancet, 396(10255), 887897.https://doi.org/10.1016/S0140-6736(20)318663

Denis Logunov, Y., Inna Dolzhikova, V., Dmitry Shcheblyakov, V., Amir Tukhvatulin, I., Olga VZubkova, V., et al. (2021). Safety and efficacy of a rAd26 and rAd5 vector-based heterologous primeboost COVID-19 vaccine: an interim analysis of a randomised controlled phase 3 trial in Russia. The Lancet, 397, 671-681.https://doi.org/10.1016/ S0140-6736(21)00234-8

Dr. Reddy's SPUTNIK V Gam-COVID-Vac. (2021, April 1). Summary of Product Characteristics. Retrieved August 28, 2021, from https://cdsco.gov.in/opencms/resources/UploadCD
SCOWeb/2018/UploadSmPC/SMPCsputinikdr.Red dys.pdf

Edward Walsh, E., Robert, F., Ann Falsey, R., Nicholas, K., Judith, A., et al. (2020). RNA-Based COVID-19 Vaccine BNT162b2 Selected for a Pivotal Efficacy Study. The New England Journal of Medicine, 383, 2439-2450. https://doi.org/10.1056/NEJMoa2027906

EMA AstraZeneca. (2021, January 29). Report onAstraZeneca, COVID 19 Vaccine (ChAdOx1 S [recombinant]). Retrieved August 29, 2021, from https://www.ema.europa.eu/en/documents/productinformation/covid-19-vaccine-astrazeneca-productinformation-approved-chmp-29-january-2021pending-endorsement en.pdf

EMA Vaxzevria. (2021, August). Report on Vaxzevria COVID-19 Vaccine (ChAdOx1-S [recombinant]). Retrieved August 29, 2021, from https://www.ema.europa.eu/en/documents/productinformation/vaxzevria-previously-covid-19-vaccineastrazeneca-epar-product-information en.pdf

Fernando Polack, P., Stephen Thomas, J., Nicholas, K., Judith, A., Alejandra, G., et al. (2020). Safety and Efficacy of the BNT162b2 mRNA Covid19 Vaccine. The New England Journal of Medicine, 383(27), 2603-2615. https://doi.org/10.1056/NEJMoa2034577

Janssen COVID-19 Vaccine (2021, August 27). Fact Sheet for Health Care Providers. Emergency Use Authorization (EUA) of the Janssen COVID-19 vaccine to prevent Coronavirus Disease 2019 (COVID-19). Retrieved September 1, 2021, from https://www.fda.gov/media/146304/download

Jerald, S., Glenda, G., Vandebosch, A., Vicky, C., Georgi, S., et al. (2021). Safety and Efficacy of Single-Dose Ad26.COV2.S Vaccine against Covid19. The New England Journal of Medicine, 384, 2187-2201.https://doi.org/10.1056/NEJMoa2101544

Jerald, S., Mathieu, L. G., Georgi, S., Dirk, H., Carla, T., et al. (2021). Interim Results of a Phase 1-2a Trial of Ad26.COV2.S Covid-19 Vaccine. The New England Journal of Medicine, 384, 1824-1835. https://doi.org/10.1056/NEJMoa2034201

Lisa Jackson, A., Evan Anderson, J., Nadine Rouphael, G., Paul Roberts, C., Mamodikoe, M., et al. (2020). An mRNA Vaccine against SARS-CoV-2 - Preliminary Report. The New England Journal of Medicine, 383, 1920-1931. https://doi.org/10.1056/NEJMoa2022483 
Int J Adv Life Sci Res. Volume 4(4)1-6

Merryn, V., Sue Ann, C. C., Shabir Madhi, A., Lily Weckx, Y., Pedro Folegatti, M., et al. (2021). Safety and efficacy of the ChAdOx1 nCoV-19 vaccine (AZD1222) against SARS-CoV-2: an interim analysis of four randomised controlled trials in Brazil, South Africa, and the UK. The Lancet, 397, 99-111.https://doi.org/https://doi.org/10.1016/ S0140-6736(20)32661-1

Merryn, V., Sue Ann, C. C., Shabir Madhi, A., Lily Weckx, Y., Pedro Folegatti, M., et al. (2021). Singledose administration and the influence of the timing of the booster dose on immunogenicity and efficacy of ChAdOx1 nCoV-19 (AZD1222) vaccine: a pooled analysis of four randomised trials. The Lancet, 397 , 881-891. https://doi.org/10.1016/ S01406736(21)00432-3

Mine Durusu, T., Hamdi Levent, D., Murat, A., Hatice Rahmet, G., Alpay, A., et al. (2021). Efficacy and safety of an inactivated whole-virion SARSCoV-2 vaccine (CoronaVac): interim results of a double-blind, randomised, placebo-controlled, phase 3 trial in Turkey. The Lancet, 398, 213-22. https://doi.org/10.1016/ S0140-6736(21)01429-X

Ministry of Health of the Russian Federation. (2020, December 28). Gam-COVID-Vac. Retrieved August 28, 2021, from https://www.fda.gov.ph/wpcontent/uploads/2021/03/12.-Proposed-Philippinepackage-insert-Instruction-Eng.pdf

Moderna COVID-19 Vaccine -Fact Sheet for Healthcare Providers Administering Vaccine (Vaccination Providers). (2021, August 27). Emergency Use Authorization (EUA) of the Moderna COVID-19 Vaccine to prevent Coronavirus Disease 2019 (COVID-19). Retrieved September 1, 2021, from

https://www.fda.gov/media/144637/download

Novavax. (2021). Covid-19 Vaccine. Retrieved August 25, 2021, from https://www.drugs.com/pro/covid-19-vaccinenovavax.html

Paul Heath, T., Eva Galiza, P., David Baxter, N., Marta, B., Duncan, B., et al. (2021). Safety and Efficacy of NVX-CoV2373 Covid-19 Vaccine. The New England Journal of Medicine. Advance online publication.https://doi.org/10.1056/NEJMoa2107659

Pedro Folegatti, M., Katie Ewer, J., Parvinder Aley, K., Brian, A., Stephan, B., et al. (2020). Safety and immunogenicity of the ChAdOx $1 \mathrm{nCoV}-19$ vaccine against SARS-CoV-2: a preliminary report of a phase $1 / 2$, single-blind, randomised controlled trial. The Lancet, 396, 467-478. https://doi.org/10.1016/ S0140-6736(20)31604-4

Pfizer-BioNTech COVID-19-Fact Sheet for Healthcare Providers Administering Vaccine (Vaccination Providers). (2021, September 22).
Emergency Use Authorization (EUA) of the PfizerBioNTech COVID-19 Vaccine to Prevent Coronavirus Disease 2019 (COVID-19). Retrieved September 4, 2021, from https://www.fda.gov/media/144413/download

Raches, E., Krishna Mohan, V., Harsh, J., Sai, P., Siddharth, R., et al. (2021). Safety and immunogenicity of an inactivated SARS-CoV-2 vaccine, BBV152: a double-blind, randomised, phase 1 trial. The Lancet Infectious Diseases, $21(5), \quad 637-646$. https://doi.org/10.1016/S14733099(20)30942-7

Raches, E., Siddarth, R., William, B., Varsha, P., Pragya, Y., et al. (2021). Efficacy, safety, and lot to lot immunogenicity of an inactivated SARS-CoV-2 vaccine (BBV152): a, double-blind, randomised, controlled phase 3 trial. medRxiv. Advance online publication.https://doi.org/10.1101/2021.06.30.2125 9439

Shengli, X., Yuntao, Z., Yanxia, W., Hui, W., Yunkai, Y., et al. (2020). Safety and immunogenicity of an inactivated SARS-CoV-2 vaccine, BBIBPCorV: a randomised, double-blind, placebocontrolled, phase 1/2 trial. The Lancet Infectious Disease, 21(1), 39-51. https://doi.org/10.1016/ S1473-3099(20)30831-8

Sinopharm WHO. (2021, May 7). Interim recommendations for use of the inactivated COVID19 vaccine BIBP developed by China National Biotec Group (CNBG), Interim guidance by WHO. Retrieved August 25, 2021, from https://apps.who.int/iris/bitstream/handle/10665/341 251/WHO-2019-nCoV-vaccines-SAGErecommendation-BIBP-2021.1eng.pdf? sequence $=1$ \&isAllowed $=y$

Sinovac Life Sciences Co., Ltd. (2021, February 22). Report on Evaluation of Safety, Efficacy and Quality of CoronaVac COVID-19 Vaccine (Vero Cell) Inactivated. Retrieved August 25, 2021, from https://www.fhb.gov.hk/download/our_work/health/2 01200/e_evaluation_report_CoronaVac.pdf

Taufik, M., Kevinkumar, K., Hardik, P., Sunil, S., Bhumika, S., et al. (2021). Safety and Immunogenicity of a DNA SARS-CoV-2 vaccine (ZyCoV-D): Results of an open-label, nonrandomized phase I part of phase I/II clinical study by intradermal route in healthy subjects in India. $\mathrm{E}$ Clinical Medicine, 38(101020), 1-13. https://doi.org/10.1016/j.eclinm.2021.101020

Zydus Cadila. (2021, August 20). Zydus receives EUA from DCGI for ZyCoV-D, the only needle-free COVID vaccine in the world. Press Release. Retrieved August 31, 2021, from https://www.zyduscadila.com/public/pdf/pressreleas e/Press\%20Release-Zydus-receives-EUA-fromDCGl-for-ZyCoV-D.pdf 